\title{
Automation of calibration process adopting metaheuristic optimization method
}

\author{
Pavel Koudela ${ }^{1}$, Juraj Chalmovský ${ }^{2}$ \\ Department of Geotechnics Faculty of Civil Engineering, Brno University of Technology, Brno, Czech Republic \\ E-mail: ${ }^{1}$ koudela.p@fce.vutbr.cz (correspondingauthor)
}

\begin{abstract}
Optimization procedures offer a possibility for time-effective determination of input parameters values for complex soil constitutive models. The following paper presents a combination of the metaheuristic Particle swarm optimization method (PSO) and commercially available solver based on the finite element method (FEM). After the brief theoretical description, different alternatives to the PSO method are reviewed and tested. An optimal alternative is chosen and further used. In the second part of the paper, the combination PSO - FEM is utilized for a fully automatic derivation of input parameters values for the Hardening small strain model from pressuremeter tests. Predicted pressurevolume curves from the axisymmetric FE model gradually converge towards the measured curve until the accuracy criterion is reached.
\end{abstract}

Keywords: particle swarm optimization, pressuremeter test, advanced soil constitutive model.

\section{Introduction}

Present material models offer a possibility for a more accurate prediction of soil behaviour. Determination of input parameters might be complicated by their number or sample disturbance due to soil sampling and tests preparation (Gilbert, 1992; Benz, 2007).

In order to reduce time requirements for a calibration process, optimization algorithms are increasingly being used in technical practice. In the following paper, the metaheuristic optimization method Particle Swarm Method is adopted for automatically driven calibration of material model inputs parameters based on pressuremeter tests. The combination of two pressuremeter tests performed in different depths was used, thus leading to a reduction of effects of soil disturbance due to sampling and sample preparation.

Automation of the calibration process is performed by using the combination of the metaheuristic PSO method and the axisymmetric finite element model of the pressuremeter test. Corrections of input parameters values are controlled by the PSO method in such a way that differences between the measurement and the FE prediction are being gradually reduced until the accuracy condition is met.

\section{Optimization procedure}

\section{Particle swarm optimization}

Particle swarm optimization (PSO) was developed in 1995 (Kennedy \& Eberhart, 1995; Eberhart \& Kennedy, 1995). This method is based on the social behaviour of birds or fish, which communicate during the finding of food and they are sharing the information. For this behaviour, the general term "swarm intelligence" is established. This problem can be defined as the finding of the extrema of a function. This function is called an objective function for the optimization procedures.

PSO uses an iterative process for finding the position of the objective function extreme. In each iteration, a new position of the particle is obtained according to (1a) consisting of three parts. In the first part, $\omega \boldsymbol{v}_{i}^{t-1}$, an inertia effect considering the particle velocity from the last iteration $v_{i}^{t-1}$ factored by the inertia weight $\omega$ is involved. The second part $c_{1} \epsilon_{1} \cdot\left(\boldsymbol{p}_{i}-\boldsymbol{x}_{i}^{t}\right)$ reflects a memory of the best position of an individual particle $\boldsymbol{p}_{i}$ (personal best). $c_{1}$ and $\epsilon_{1}$ are the acceleration constant and the vector of random numbers (in range of 0 and 1), respectively. Finally, the third 
part $c_{2} \epsilon_{2} \cdot\left(\boldsymbol{g}^{*}-\boldsymbol{x}_{i}^{t}\right)$ contains a collective memory, which is represented by the global best position $\boldsymbol{g}^{*}$ for a whole group of particles. $c_{2}$ and $\epsilon_{2}$ are the acceleration constant and the vector of random numbers (in range of 0 and 1), respectively. A movement of a particle (a change of coordinates in a searching space) is controlled via its velocity in the search space (Figure 1). A new position $\boldsymbol{x}_{i}^{t+1}$ of a particle, $i$ in a current time step $t$ is given by the previous position $\boldsymbol{x}_{i}^{t}$ and the current velocity $\boldsymbol{v}_{i}^{t+1}$ according to (1b).

$$
\begin{gathered}
\boldsymbol{v}_{i}^{t+1}=\omega \boldsymbol{v}_{i}^{t}+c_{1} \epsilon_{1} \cdot\left(\boldsymbol{p}_{i}-\boldsymbol{x}_{i}^{t}\right)+c_{2} \epsilon_{2} \cdot\left(\boldsymbol{g}^{*}-\boldsymbol{x}_{i}^{t}\right) \\
\boldsymbol{x}_{i}^{t+1}=\boldsymbol{x}_{i}^{t}+\boldsymbol{v}_{i}^{t+1} .
\end{gathered}
$$

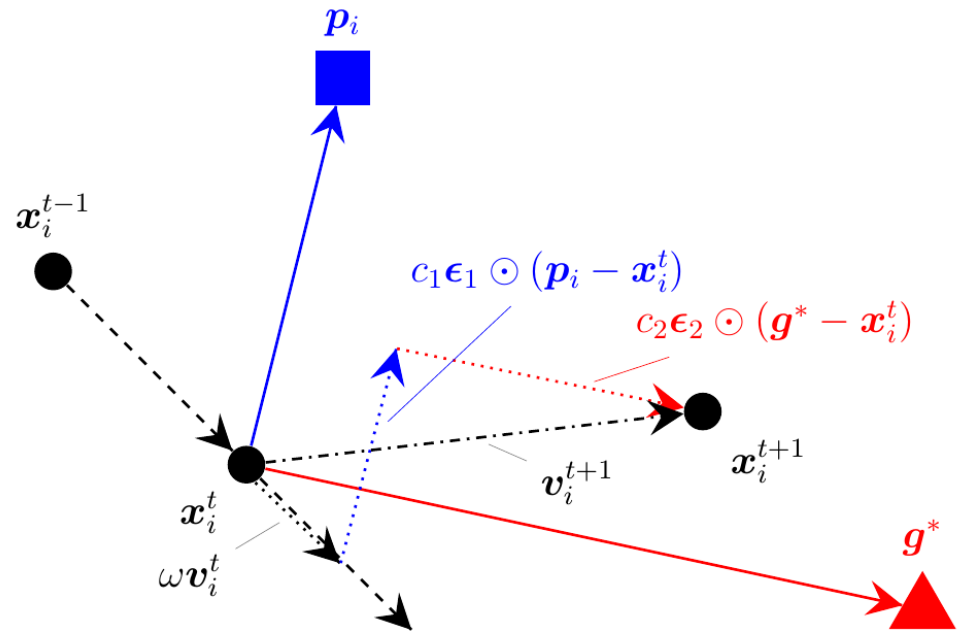

Figure 1. Movement of one particle during the iterative process of the PSO

\section{Summary of various adjustments of PSO}

Several modifications of the original PSO method (Shi \& Eberhart, 1998; Heris, 2016; Yang, 2008) have been proposed since its development (Kennedy \& Eberhart, 1995; Eberhart \& Kennedy, 1995). Seven main adjustments summarized in Table 1 and briefly introduced in this chapter were therefore tested and compared. Based on this review, the most appropriate alternative was chosen for further application to a geotechnical boundary value problem.

Table 1. Summary of tested PSO alternatives

\begin{tabular}{|c|c|c|c|c|c|}
\hline $\begin{array}{c}\text { Adjustment } \\
\text { No. }\end{array}$ & New particle velocity equation & $c_{1}$ & $c_{2}$ & $\omega$ & $\omega_{\text {damp }}$ \\
\hline 02 & $\boldsymbol{v}_{\boldsymbol{i}}^{\boldsymbol{t}}=\boldsymbol{v}_{\boldsymbol{i}}^{\boldsymbol{t}-1}+c_{1} \epsilon_{1} \cdot\left(\boldsymbol{p}_{\boldsymbol{i}}-\boldsymbol{x}_{\boldsymbol{i}}^{\boldsymbol{t}-1}\right)+c_{2} \epsilon_{2} \cdot\left(\boldsymbol{g}^{*}-\boldsymbol{x}_{\boldsymbol{i}}^{\boldsymbol{t}-1}\right)$ & 2 & 2 & - & - \\
\hline 03 & $\boldsymbol{v}_{\boldsymbol{i}}^{\boldsymbol{t}}=\omega \boldsymbol{v}_{\boldsymbol{i}}^{\boldsymbol{t}-1}+c_{1} \epsilon_{1} \cdot\left(\boldsymbol{p}_{\boldsymbol{i}}-\boldsymbol{x}_{\boldsymbol{i}}^{\boldsymbol{t}-1}\right)+c_{2} \epsilon_{2} \cdot\left(\boldsymbol{g}^{*}-\boldsymbol{x}_{\boldsymbol{i}}^{\boldsymbol{t}-1}\right)$ & 1.49618 & 1.49618 & 0.7298 & - \\
\hline 04 & $\boldsymbol{v}_{\boldsymbol{i}}^{\boldsymbol{t}}=\omega \boldsymbol{v}_{\boldsymbol{i}}^{\boldsymbol{t}-1}+c_{1} \epsilon_{1} \cdot\left(\boldsymbol{p}_{\boldsymbol{i}}-\boldsymbol{x}_{\boldsymbol{i}}^{\boldsymbol{t}-1}\right)+c_{2} \epsilon_{2} \cdot\left(\boldsymbol{g}^{*}-\boldsymbol{x}_{\boldsymbol{i}}^{\boldsymbol{t}-1}\right)$ & 2 & 2 & 1 & 0.99 \\
\hline 06 & $\boldsymbol{v}_{\boldsymbol{i}}^{\boldsymbol{t}}=\omega \boldsymbol{v}_{\boldsymbol{i}}^{\boldsymbol{t}-1}+c_{1}(\epsilon-0.5)+c_{2} \epsilon_{2} \cdot\left(\boldsymbol{g}^{*}-\boldsymbol{x}_{\boldsymbol{i}}^{\boldsymbol{t}-1}\right)$ & 0.2 & 0.5 & 1 & 0.99 \\
\hline 08 & $\boldsymbol{v}_{\boldsymbol{i}}^{\boldsymbol{t}}=\omega \boldsymbol{v}_{\boldsymbol{i}}^{\boldsymbol{t}-1}+c_{1}(\epsilon-0.5)+c_{2} \epsilon_{2} \cdot\left(\boldsymbol{g}^{*}-\boldsymbol{x}_{\boldsymbol{i}}^{\boldsymbol{t}-1}\right)$ & $0.7^{t}$ & 0.5 & 1 & 0.99 \\
\hline 09 & $\boldsymbol{v}_{\boldsymbol{i}}^{\boldsymbol{t}}=\omega \boldsymbol{v}_{\boldsymbol{i}}^{\boldsymbol{t}-1}+c_{1} \epsilon_{1} \cdot\left(\boldsymbol{p}_{\boldsymbol{i}}-\boldsymbol{x}_{\boldsymbol{i}}^{\boldsymbol{t}-1}\right)+c_{2} \epsilon_{2} \cdot\left(\boldsymbol{g}^{*}-\boldsymbol{x}_{\boldsymbol{i}}^{\boldsymbol{t}-1}\right)$ & $0.7^{t}$ & 0.5 & 1 & 0.99 \\
\hline 34 & $\boldsymbol{v}_{\boldsymbol{i}}^{\boldsymbol{t}}=\omega \boldsymbol{v}_{\boldsymbol{i}}^{\boldsymbol{t}-1}+c_{1} \epsilon_{1} \cdot\left(\boldsymbol{p}_{\boldsymbol{i}}-\boldsymbol{x}_{\boldsymbol{i}}^{\boldsymbol{t}-1}\right)+c_{2} \epsilon_{2} \cdot\left(\boldsymbol{g}^{*}-\boldsymbol{x}_{\boldsymbol{i}}^{\boldsymbol{t}-1}\right)$ & 1.49618 & 1.49618 & 0.7298 & 0.99 \\
\hline
\end{tabular}


(Kennedy \& Eberhart, 1995; Eberhart \& Kennedy, 1995) recommended $\omega=1$ and $c_{1}=c_{2}=2$. These values are used in the adjustment " 02 ". Furthermore, pre-chosen boundary limits of particle positions were applied.

The study (Shi \& Eberhart, 1998) of an inertia weight provides recommended values of all constants $\omega=0.7298$ and $c_{1}=c_{2}=1.49618$. The constriction factor $\chi(2)$ is involved in the particle velocity calculation (3). The value of parameter $\varphi$ is recommended as 4.1 leading to the constriction factor value 0.7298 . This alternative is called " 03 ".

$$
\begin{gathered}
\chi=\frac{2}{\left|2-\varphi-\sqrt{\varphi^{2}-4 \varphi}\right|} ; \\
\varphi=c_{1}+c_{2}, \varphi>4 ; \\
\boldsymbol{v}_{i}^{t}=\chi\left(\omega \boldsymbol{v}_{i}^{t-1}+c_{1} \epsilon_{1} \cdot\left(\boldsymbol{p}_{i}-\boldsymbol{x}_{i}^{t-1}\right)+c_{2} \epsilon_{2} \cdot\left(\boldsymbol{g}^{*}-\boldsymbol{x}_{i}^{t-1}\right)\right) .
\end{gathered}
$$

In order to reduce swarm position instability, an inertia damping was proposed by (Heris, 2016). Damping is defined by (4) in which the damping coefficient $\omega_{\text {damp }}=0.99$. Damping is used in the alternative " 04 ".

$$
\omega^{t}=\omega^{t-1} \cdot \omega_{\text {damp }} .
$$

In the so-called accelerated approach proposed by (Yang, 2008), the local best term $c_{1} \epsilon_{1} \cdot\left(\boldsymbol{p}_{\boldsymbol{i}}-\boldsymbol{x}_{\boldsymbol{i}}^{\boldsymbol{t}-1}\right)$ is replaced by the random vector $c_{1}(\epsilon-0,5)$. The best position of the individual particle is therefore not considered. This approach is tested as the alternative " 06 ".

Next modification is based on a similar approach as the previous alternative. Moreover the influence of the random vector $c_{1}(\epsilon-0,5)$ decreases with time $t$ (number of iterations) as the coefficient $c_{1}$ equals to $0.7^{t}$. This modification is called " 08 ".

The local best position and validity of the (1) is preserved in the adjustment "09". Exponential damping and $c_{1}$, $c_{2}$ values are however taken over from the previous adjustment.

Finally, the alternative " 34 " presents a combination of the modifications " 03 " and " 04 ". In this way a positive effect of the inertia damping and its exponential decrease (inertia weight $\omega=0.7298, \omega_{\text {damp }}=0.99$ ) and recommended values of $c_{1}$ and $c_{2}(1.49618)$ are used in one alternative.

\section{Testing of various adjustments of PSO}

The efficiency of each modification was analyzed by means of finding a global extreme of chosen testing functions (Molga \& Smutnicki, 2005). These functions are mostly multimodal, two or multi-dimensional with several local extremes. MATLAB script involving the PSO method and a library of testing functions was created for this purpose. The script run was repeated 100 times for each combination of the particular PSO modification and testing function. A normalized mean distance of particles from the global extreme coordinates was then evaluated (5).

$$
s_{\text {norm }}=\frac{\sum_{j=1}^{r} \frac{\sum_{i=1}^{n} \frac{s_{\text {particle }}}{s_{\max }}}{n}}{r},
$$

where $s_{\text {particle }}$ is the actual particle distance from a global minima of a testing function, $s_{\max }$ is the maximal possible distance of a particle in a search space, $n$ is the number of particles, $r$ is the number of repetitions.

Results for six different testing functions are shown in Figure 2 for 50 iteration and 10 particles. Adjustment " 02 " neglecting particles damping in successive iterations showed the worst results. Other adjustments, which involving more sophisticated control of particles movements, reached better results during the optimization for all testing functions. The sum mean distances values over all testing function was the lowest at adjustment " 34 " of all considered adjustments. Due to this, in further application to a boundary value problem adjustment " 34 " was involved. 


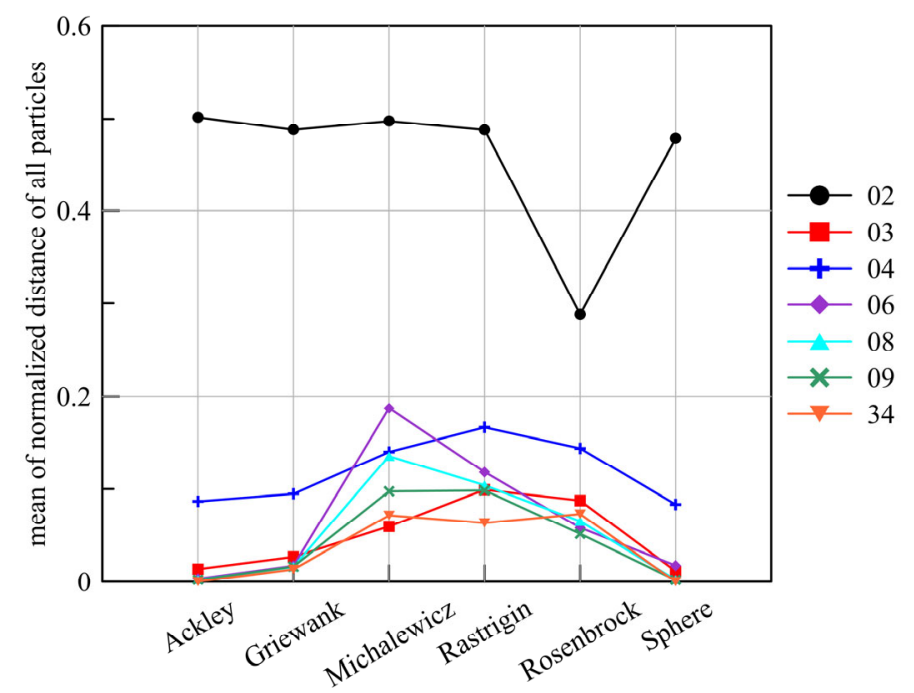

Figure 2. Plot of the normalized mean distance of all particle on the end of PSO runs

\section{Definition of geotechnical problem}

The initial position of the particle $i$ during the iteration $t$ in three dimensional space is defined by the coordinates $\boldsymbol{x}_{i}^{t}=\left\{p_{i}, q_{i}, r_{i}\right\}$. Consequently, the objective function value in this particular position is $f\left(p_{i}, q_{i}, r_{i}\right)$. The PSO method governs the particle position update in the subsequent iteration $\boldsymbol{x}_{i}^{t+1}$. The particle position is being corrected until the coordinates of the global extreme $\boldsymbol{G}^{*}$ are reached (see Figure 3a). In the current problem, particle coordinates are represented by constitutive model input parameters: e. g. $E^{\prime}, c^{\prime}, \varphi^{\prime}$ for the Mohr - Coulomb model (see Figure 3b). Predicted load - displacement curves (or another response of geotechnical construction) for two sets of input parameters values in subsequent iterations are shown in Figure 3c. The red curve represents a measurement to which a prediction is approaching in subsequent iterations.

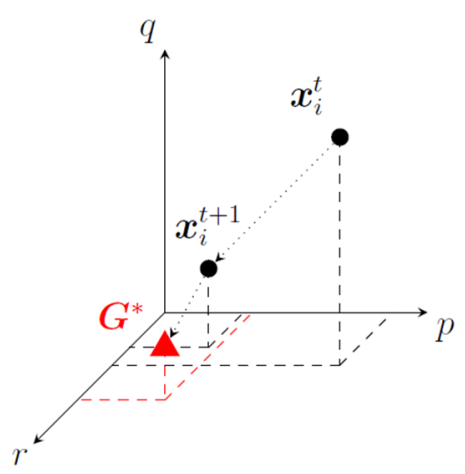

a)

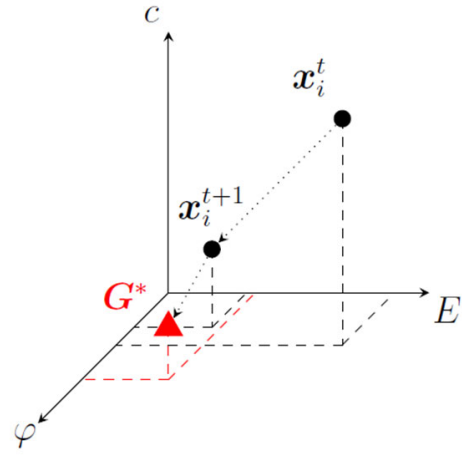

b)

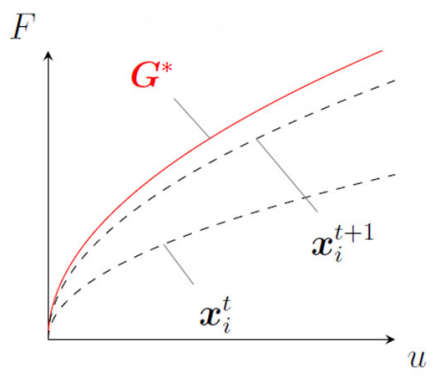

c)

Figure 3. Particle positions in two spaces and predicted the load-displacement curve

\section{Application to boundary value problem}

For the determination of input parameters values of chosen constitutive models, the combination of two pressuremeter tests in different depths $(4.5 \mathrm{~m}$ and $9.5 \mathrm{~m})$ is used. The pressuremeter test was performed in Neogene clay (Miocene) in the city Brno, Czech Republic. The Neogene clay is classified as Cl (ISO 14689) with gravimetric water content of $32 \%-37 \%$, plasticity index of $29 \%-33.4 \%$ and liquidity index of $77 \%-85 \%$ (Miča, Chalmovský, Fiala, \& Račanský, 2011). The optimization application created for this application involving the PSO method and FEM solver.

\section{Description of the numerical model}

The axisymmetric FE model of the pressuremeter test was prepared in Plaxis 2D 2016 (see Figure 4). The height of the model is dependent on the number of pressuremeter and the width of the model is the same for either pressuremeters. The boundaries are chosen such that their influence is negligible. The pressure to the cavity is simulated by the line load. 


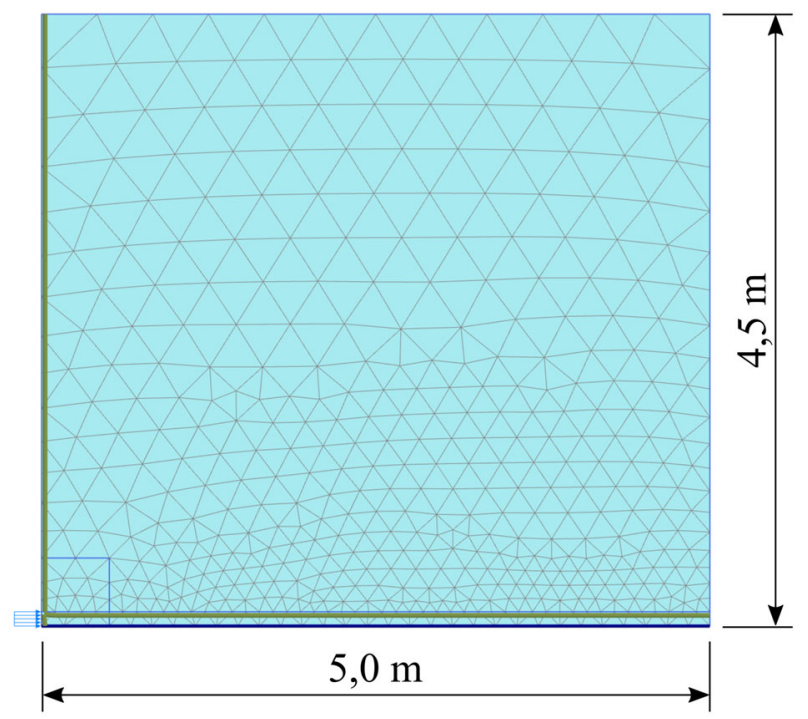

Figure 4. Axisymmetric FE model of the pressuremeter test

Nodal displacements obtained from the numerical model are transformed into the respective volume changes of the pressuremeter cell (6) (Brinkgreve, Kumarswamy, \& Swolfs, 2016) assuming a cylindrical expansion of the cell.

$$
\frac{\Delta V}{V_{0}}=\frac{\left(R_{0}+u_{x}\right)^{2}-R_{0}^{2}}{R_{0}^{2}}
$$

where: $\Delta V$ is the increment of pressuremeter cell volume, $V_{0}$ is the initial volume of the pressuremeter cell, $R_{0}$ is the initial radius of the pressuremeter cell and $u_{x}$ is the horizontal (radial) displacement predicted by the numerical model.

The optimization algorithm minimizes the objective function value defined according to (7). In the objective function, both pressuremeter tests are included.

$$
f(\boldsymbol{x})=\sum_{j=1}^{k} \sum_{i=1}^{n}\left(V_{j i}^{m e a s}-V_{j i}^{f e m}\right)^{2},
$$

where $V_{j i}^{\text {meas }}$ is the measured volume during the pressuremeter test, $V_{j i}^{f e m}$ is the predicted volume obtained from the numerical model, $i$ is the loading stage (according to the pressuremeter test), $j$ is the number of the pressumeter test.

Three material models were used during the analysis. Mohr-Coulomb (MC) model is a well-known linear elastic perfectly-plastic model. Hardening soil (HS) model is the elastoplastic model considering shear and compression hardening (Schanz, Vermeer, \& Bonnier, 1999). Hardening soil small strain (HSS) model is the extension of the Hardening soil model and incorporates strain dependent shear moduli in small strains (Benz, 2007).

\section{Sensitivity analysis}

In order to identify governing parameters and thus reduce the number of optimized variables, the sensitivity analysis was first carried out. Relative sensitivity (Brinkgreve et al., 2016) of the horizontal (radial) displacement in the middle of the cylindrical cavity on the respective input variable change was investigated. In the case of all three material models both soil stiffness and strength have an important role (see Figure 5a, Figure 5b, Figure 5c). This is due to the fact, that the failure criterion is reached in the vicinity of the cylindrical cavity. Shear strength is governed by the effective friction angle $\varphi^{\prime}$. In the case of the HS model, the unloading-reloading modulus $E_{u r}^{r e f}$ has more influence than the remaining stiffness parameters (see Figure 5b). This is due to the fact the overconsolidation of Brno clay is considered in the numerical analysis, thus enlarging the stress range in the unloading - reloading regime. In the case of HSS model, both the small strain shear modulus $G_{0}^{r e f}$ and the unloading - reloading modulus $E_{u r}^{r e f}$ reached the relative sensitivity values above $10 \%$ (see Figure $5 \mathrm{c}$ ). 


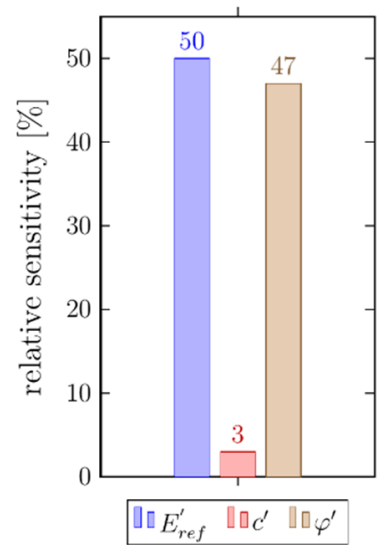

a) MC model

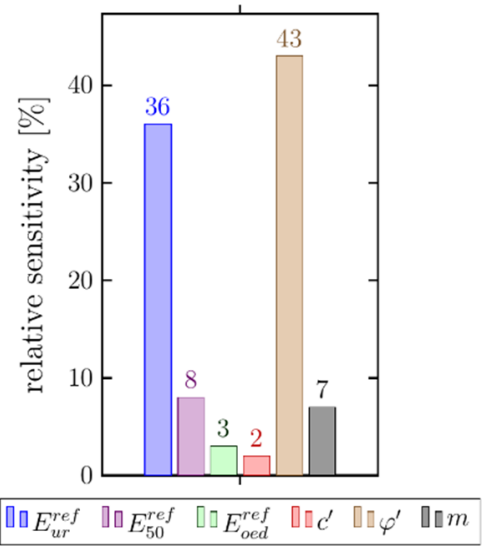

b) HS model

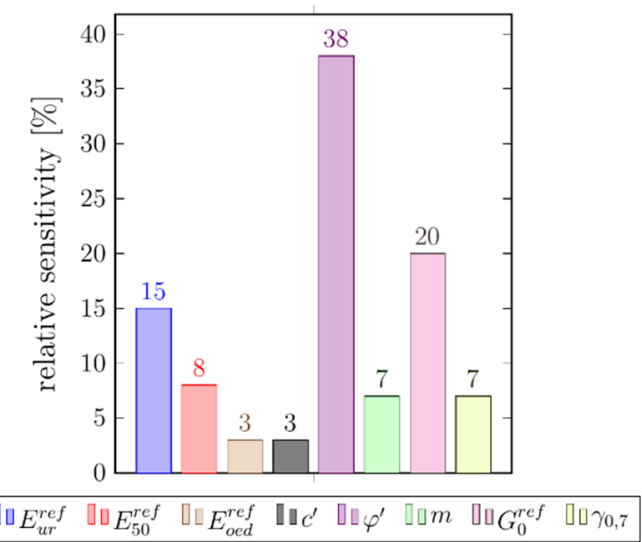

c) HSS model

Figure 5. Sensitivity analysis

\section{Optimization}

In this section, only the optimization process of the HSS model input parameters is presented. Initial values of input parameters are summarized in Table 2. The values of $E_{50}^{\text {ref }}$ and $E_{\text {oed }}^{\text {ref }}$ are taken as $E_{50}^{\text {ref }}=E_{\text {oed }}^{\text {ref }}=E_{u r}^{\text {ref }} / 3$. Considering the sensitivity analysis results, the variables $E_{u r}^{r e f}, \varphi^{\prime}$ and $G_{0}^{r e f}$ were further optimized.

Table 2. Initial parameters of HSS model

\begin{tabular}{|c|c|c|c|c|c|c|c|c|}
\hline & $\begin{array}{c}E_{u r}^{r e f} \\
{[\mathrm{kPa}]}\end{array}$ & $\begin{array}{c}E_{50}^{r e f} \\
{[\mathrm{kPa}]}\end{array}$ & $\begin{array}{c}E_{\text {oed }}^{r e f} \\
{[\mathrm{kPa}]}\end{array}$ & $\begin{array}{c}c^{\prime} \\
{[\mathrm{kPa}]}\end{array}$ & $\begin{array}{c}\varphi^{\prime} \\
{\left[{ }^{\circ}\right]}\end{array}$ & $\begin{array}{c}m \\
{[-]}\end{array}$ & $\begin{array}{c}G_{0}^{r e f} \\
{[\mathrm{kPa}]}\end{array}$ & $\gamma_{0,7}$ \\
\hline initial values of parameter & 18000 & 6000 & 6000 & 6 & 20 & 0.5 & 30000 & $1 e^{-4}$ \\
\hline
\end{tabular}

In order to keep the computational time within acceptable limits, a combination of 5 particles in the swarm and 20 iterations was used. In the course of calculations with this combination of particles and iterations, the objective function maintains the same value during at least 5 iterations before the final iteration was completed.

The optimization algorithm generates random values of input parameters for all particles in the first step except the first particle where parameters are defined according to Table 2. After that, the optimization continues as described in the sections above. The measured pressuremeter curve, initial prediction based on variable values summarized in Table 2 and predictions for five different iterations are shown in Figure 6 for the pressuremeter test conducted in depth of $4.5 \mathrm{~m}$.

The optimized values of input parameters are summarized in Table 3 . The measured pressuremeter curves, initial and final predictions in case of simultaneous optimization of both tests are shown in Figure 7. Final stiffness parameters are significantly higher than the initial estimates. Obtained values are in reasonable agreement with those published in (Miča et al., 2011) (see Table 4). These values were derived from the numerical back - analysis of deep excavations in similar geological conditions. The obtained values of unloading - reloading modulus $E_{u r}^{\text {ref }}$ and friction angle $\varphi^{\prime}$ are slightly higher, however, the small strain shear modulus $G_{0}^{\text {ref }}$ is slightly lower compared to the data published in (Miča et al., 2011).

Table 3. Results of optimization of input parameters of the HSS model

\begin{tabular}{|l|c|c|c|c|}
\hline & $\begin{array}{c}E_{u r}^{r e f} \\
{[\mathrm{kPa}]}\end{array}$ & $\begin{array}{c}\varphi^{\prime} \\
{\left[{ }^{\circ}\right]}\end{array}$ & $\begin{array}{c}G_{0}^{r e f} \\
{[\mathrm{kPa}]}\end{array}$ & $\begin{array}{c}f\left(\mathbf{g}^{*}\right) \\
{\left[\mathrm{cm}^{6}\right]}\end{array}$ \\
\hline optimized value of parameter & 43310 & 24.8 & 55628 & 29073 \\
\hline
\end{tabular}


Table 4. Values of parameters obtained from back - analysis of deep excavations (Miča et al., 2011)

\begin{tabular}{|c|c|c|c|}
\hline & $\begin{array}{c}E_{u r}^{r e f} \\
{[\mathrm{kPa}]}\end{array}$ & $\begin{array}{c}\varphi^{\prime} \\
{\left[{ }^{\circ}\right]}\end{array}$ & $\begin{array}{c}G_{0}^{\text {ref }} \\
{[\mathrm{kPa}]}\end{array}$ \\
\hline value of parameter & 36170 & 24 & 60000 \\
\hline
\end{tabular}

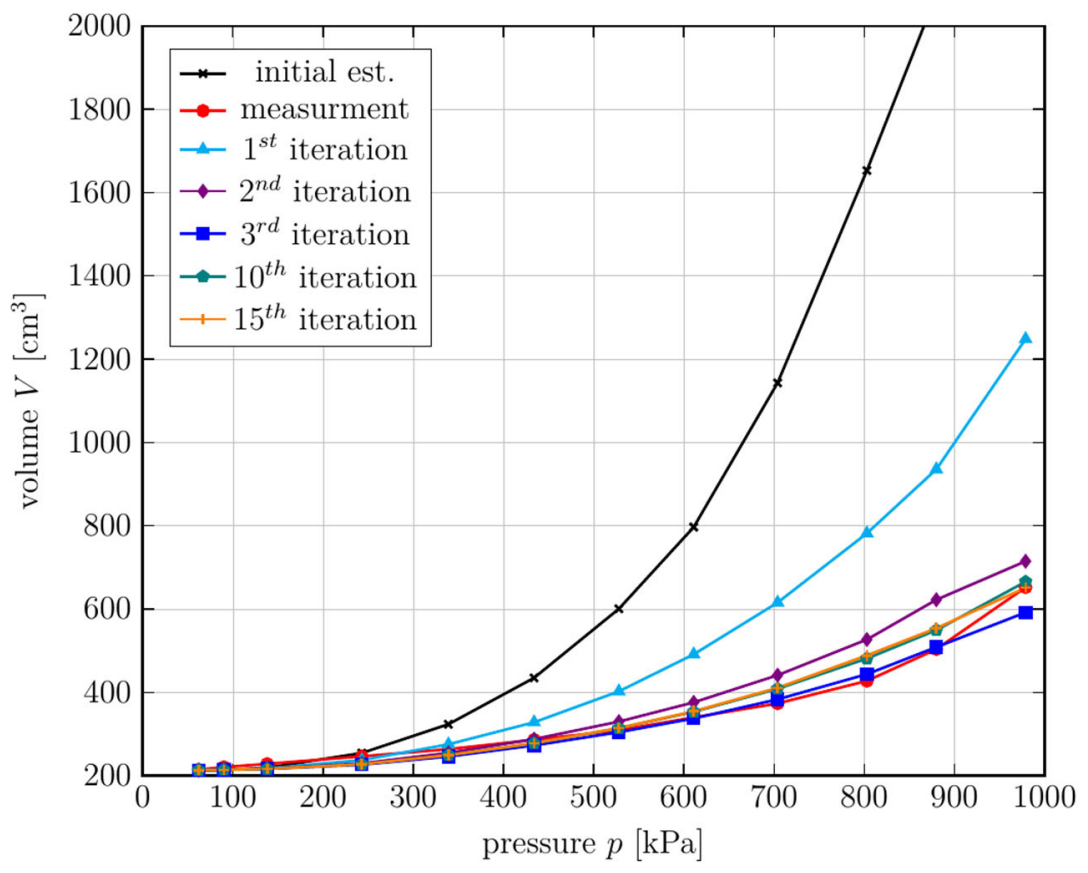

Figure 6. Step by step convergence of predicted pressuremeter curves

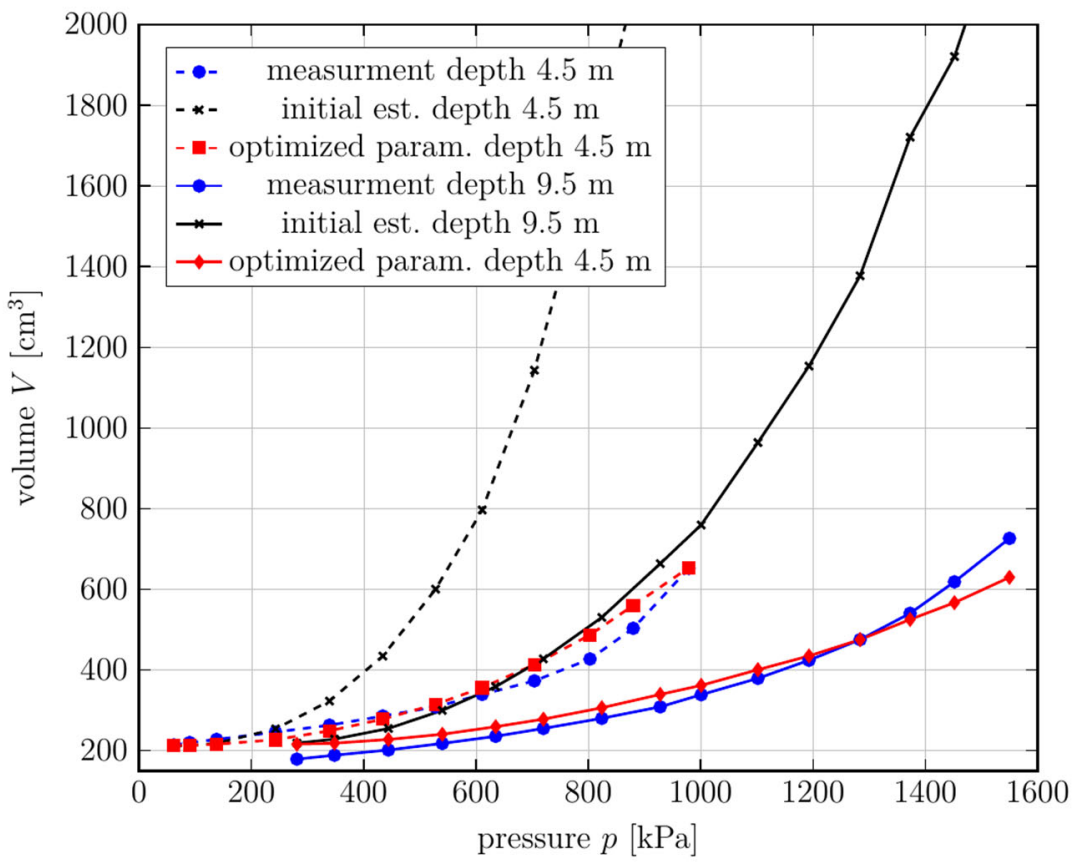

Figure 7. Results of optimization - measured pressuremeter curves, predicted curves with initial parameters and pressuremeter curves with optimization parameters 


\section{Conclusions}

The results presented in this paper confirmed that the combination of the metaheuristic optimization technique and the finite element solver might be a useful and effective way for determination of input parameters of complex soil constitutive models based on in-situ tests.

Usage of the optimized values of input parameters led to the simultaneous acceptable agreement with both pressuremeter tests from different depths. At the same time, these values are comparable with literature data obtained from a different type of constructions (retaining structures).

Further research is currently mainly focused on the following areas: ensuring uniqueness and repeatability when applying stochastic optimization methods, multi-objective optimization, comparison with other metaheuristic methods and application of these methods on monitored geotechnical constructions.

\section{Acknowledgements}

This paper has been worked out under the project "AdMaS UP - Advanced Materials, Structures and Technologies" [grant number LO1408], supported by Ministry of Education, Youth and Sports under the "National Sustainability Programme I" and internal research grant of Brno University of Technology [grant number FAST-S-18-5356].

\section{References}

Benz, T. (2007). Small-strain stiffness of soils and its numerical consequences. Stuttgart: Inst. für Geotechnik.

Brinkgreve, R. B. J., Kumarswamy, S., \& Swolfs, W. M. (2016). Plaxis: finite element code for soil and rock analyses: 2D-version [user's guide]. Delft, Netherlands.

Eberhart, R., \& Kennedy, J. (1995). A new optimizer using particle swarm theory. In MHS'95. Proceedings of the Sixth International Symposium on Micro Machine and Human Science (pp. 39-43). Piscataway, NJ: IEEE. https://doi.org/10.1109/MHS.1995.494215

Gilbert, P. A. (1992). Effect of sampling disturbance on laboratory-measured soil properties. Vicksburg.

Heris, M. K. (2016). Video tutorial of PSO implementation in MATLAB. Yarpiz - Academic Source Codes and Tutorials. Retrieved from http://yarpiz.com/440/ytea101-particle-swarm-optimization-pso-inmatlab-video-tutorial

Kennedy, J., \& Eberhart, R. (1995). Particle swarm optimization. In Proceedings of ICNN'95 - International Conference on Neural Networks (pp. 1942-1948). IEEE. https://doi.org/10.1109/ICNN.1995.488968

Miča, L., Chalmovský, J., Fiala, R., \& Račanský, V. (2011). Numerická analýza pažení stavebních jam. Brno: Akademické nakladatelství CERM.

Molga, M., \& Smutnicki, C. (2005). Test functions for optimization needs. Retrieved from https://www.robertmarks.org/Classes/ENGR5358/Papers/functions.pdf

Schanz, T., Vermeer, P. A., \& Bonnier, P. G. (1999). The hardening soil model: Formulation and verification. In R. B. J. Brinkgreve (Ed.), Beyond 2000 in Computational Geotechnics (pp. 281-296). Taylor \& Francis. https://doi.org/10.1201/9781315138206-27

Shi, Y., \& Eberhart, R. (1998). A modified particle swarm optimizer. In 1998 IEEE International Conference on Evolutionary Computation Proceedings. IEEE World Congress on Computational Intelligence (Cat. No. 98TH8360) (pp. 69-73). IEEE. https://doi.org/10.1109/ICEC.1998.699146

Yang, X.-S. (2008). Introduction to mathematical optimization: from linear programming to metaheuristics. Cambridge, UK: Cambridge International Science Publishing. 